\title{
Vulnérabilité et dynamiques adaptatives des agropasteurs aux mutations climatiques dans la commune de Tchaourou au Bénin
}

\author{
Gildas Louis Djohy ${ }^{1 *}$ Boni Sounon Bouko ${ }^{1}$
}

\section{Mots-clés}

Bovin, vulnérabilité, adaptation aux changements climatiques, Bénin

Submitted: 21 February 2020

Accepted: 13 October 2020

Published: 1 March 2021

DOI: $10.19182 /$ remvt.3619

\section{Résumé}

L'élevage bovin est la principale activité économique des communautés peules du Nord Centre Bénin. Cette activité dans la commune de Tchaourou, au climat sud-soudanien à une saison pluvieuse et une saison sèche, est très vulnérable aux effets désastreux des mutations climatiques. La présente étude a eu pour objectif d'appréhender les perceptions des agropasteurs sur les mutations climatiques, et d'analyser leur vulnérabilité et leurs stratégies d'adaptation pour y faire face. L'approche méthodologique s'est reposée sur la collecte de données qualitatives socioanthropologiques issues d'enquêtes de terrain. Les données des entretiens individuels, des groupes de discussion et des observations directes ont permis de générer des matrices de sensibilité et de vulnérabilité des ressources et des agropasteurs, et d'analyser les pratiques adaptatives de ces derniers. Les résultats ont montré une grande similitude des manifestations des mutations climatiques perçues par les agroéleveurs. Ces manifestations étaient entre autres la hausse des températures, le prolongement de la saison sèche, la fin précoce de la saison des pluies et la baisse pluviométrique. Ces risques climatiques induisaient l'assèchement précoce des points d'eau, l'amenuisement du disponible fourrager et la dégradation de l'état sanitaire des animaux par le développement de pathologies. Face à cette instabilité du climat et à ses effets, les agropasteurs ont développé diverses stratégies pour en atténuer les impacts. Ces stratégies ont montré une performance variée selon leur efficacité et méritent d'être renforcées par une politique sectorielle sensible aux risques climatiques.

- Comment citer cet article : Djohy G.L., Sounon Bouko B., 2021. Vulnerability and adaptive dynamics of agropastoralists to climate change in the commune of Tchaourou in Benin. Rev.Elev.Med. Vet. Pays Trop., 74 (1): 27-35, doi: 10.19182/remvt.36319

\section{INTRODUCTION}

L’Afrique est considérée comme la région la plus vulnérable aux effets dévastateurs des changements climatiques (GIEC, 2001 ; CSAO/OCDE, 2008) et aucune partie du continent africain n'est épargnée. Toutes les régions du Bénin sont diversement touchées par les mutations climatiques (Hounkponou et al., 2009 ; Boko et al., 2012). Le Nord Bénin en général et la commune de Tchaourou en particulier sont affectés par la dégradation des conditions climatiques et leurs

1. Département de géographie et aménagement du territoire (DGAT), Université de Parakou, 03 BP 303 Parakou, Bénin.

* Auteur pour la correspondance

Email : gildasdjohy@gmail.com effets induits. L'évolution du climat est particulièrement caractérisée par la baisse et la mauvaise répartition spatio-temporelle des précipitations, la modification des régimes pluviométriques, l'augmentation de la température, l'accroissement de la durée des périodes sèches et les pluies périodiques (Boko et al., 2012). Le potentiel de croissance de l'économie béninoise en général et des communes en particulier se trouve ainsi menacé par les nouvelles conditions climatiques. Il dépend en majorité de la production agricole et de l'élevage qui sont tributaires des précipitations. Ainsi, la variabilité des conditions climatiques a rendu plus difficile la gestion des ressources en eau pour l'abreuvement du bétail et a entraîné une restriction du disponible fourrager aussi bien en quantité qu'en qualité (Totin Vodounon et al., 2016). La production animale notamment bovine, deuxième activité économique après l'agriculture au Bénin, particulièrement dans le département du Borgou (De Haan et al., 1997), s'en trouve drastiquement affectée. Ce phénomène conduit les pasteurs à manquer d'eau 
et de fourrages devenus de plus en plus difficiles à trouver (Djohy, 2015). Cet article vise, d'une part, à appréhender les perceptions des agropasteurs sur les mutations climatiques et, d'autre part, à analyser leur vulnérabilité et leurs stratégies d'adaptation pour faire face aux nouvelles conditions climatiques.

\section{MATERIEL ET METHODES}

\section{Milieu d'étude}

Létude a été conduite dans la commune de Tchaourou, située dans le département du Borgou. Elle s'étend sur 7256 kilomètres carrés, soit $28 \%$ de la superficie du département et environ 6,5\% du territoire national (PDC, 2017). Tchaourou est limitée au nord par les communes de Parakou et N'Dali, au nord-est par celle de Pèrèrè, au sud par celle de Ouèssè, à l'ouest par celles de Bassila et Djougou, et à l'est par le Nigeria. Elle est située entre $8^{\circ} 46^{\prime}$ et $9^{\circ} 42^{\prime} \mathrm{N}$, et $1^{\circ} 58^{\prime}$ et $3^{\circ} 10^{\prime} \mathrm{E}$ (figure 1).

Le climat local est de type sud soudanien caractérisé par une saison pluvieuse et une saison sèche. Les moyennes pluviométriques annuelles varient entre 1100 et 1200 millimètres (Kora, 2006). Le réseau hydrographique est principalement dominé par les affluents du fleuve Ouémé et de la rivière Okpara. Ainsi, les sources d'eau de surface à Tchaourou sont composées de deux plans d'eau de $27 \mathrm{~km}$ de long, de 11 cours d'eau permanents de $295 \mathrm{~km}$ et d'un cours d'eau temporaire de $683 \mathrm{~km}$ (PDC, 2017). Ces cours et plans d'eau présentent des potentialités exploitables par les agropasteurs. Ils constituent des points d'attraction des animaux à la recherche de sites d'abreuvement et de pâturages. La commune dispose également de nombreuses retenues d'eau qui favorisent l'abreuvement des animaux en saison sèche, notamment celle de Boukousséra, de Papanè, de Kaki Koka, d'Alafiarou, de Sebou, de GahBaka, de Kpassa et de Winra (Chabi Boum, 2011 ; PDC, 2017).

Les formations végétales naturelles (forêt dense, galerie forestière, forêt claire et savane boisée, savane arborée et arbustive) fournissent aux animaux différentes espèces fourragères en saison sèche. Les forêts communales recouvrent 1725 kilomètres carrés, soit 23,8\% de la superficie de la commune (Kora, 2006 ; PDC, 2017). Les strates herbacées sont caractérisées par des espèces comme Andropogon gayanus et Imperata cylindrica. Les galeries forestières se développent le long des cours d'eau notamment dans les vallées de l'Ouémé et de l'Okpara.

Selon le quatrième Recensement général de la population et de l'habitation (RGPH-4), la population de Tchaourou a augmenté considérablement avec 223138 habitants en 2013 contre 106852 en 2002. Cette population est composée principalement de Bariba (34,2\%), de Peuls (18,9\%), de Nagot (15,8\%), d'Otamari (12,9\%) et de Yom-Lokpa (10,9\%), qui sont à la fois agriculteurs et éleveurs (Kora, 2006). La densité moyenne de la population est passée de 15 habitants $/ \mathrm{km}^{2}$ en 2002 à 31 en 2013. Plus de $90 \%$ de la population pratique l'agriculture (Kora, 2006), notamment les cultures céréalières (maïs, sorgho, riz), les légumineuses (niébé, soja, arachide), les racines et tubercules (igname, manioc), les maraîchères (piment, tomate, gombo), et les cultures de rente (coton, anacarde, karité).

L'élevage pratiqué est essentiellement extensif et constitue la principale activité des Peuls et la seconde activité des autres groupes socioculturels. Selon le Secteur communal de développement agricole de Tchaourou, le cheptel communal comprend diverses espèces : 40372 bovins, 11755 ovins et 14093 caprins en 2016 (PDC, 2017). Les animaux sont menacés par diverses pathologies, dont la fièvre aphteuse, la péripneumonie contagieuse bovine, la pasteurellose bovine, la trypanosomose, la brucellose et la peste des petits ruminants (Chabi Boum, 2011). Les éleveurs peuls de la commune cultivent principalement du maïs, du mil/sorgho et de l'igname pour assurer non seulement leur autosuffisance alimentaire mais également pour nourrir le bétail à partir des résidus de cultures. En rupture avec le mode d'élevage ancestral, ils sont passés du système pastoral fondé sur la mobilité des animaux à un système agropastoral basé sur la complémentation alimentaire du bétail. Ainsi, ils ont recours à l'agriculture pour mieux faire face aux incertitudes climatiques et environnementales.

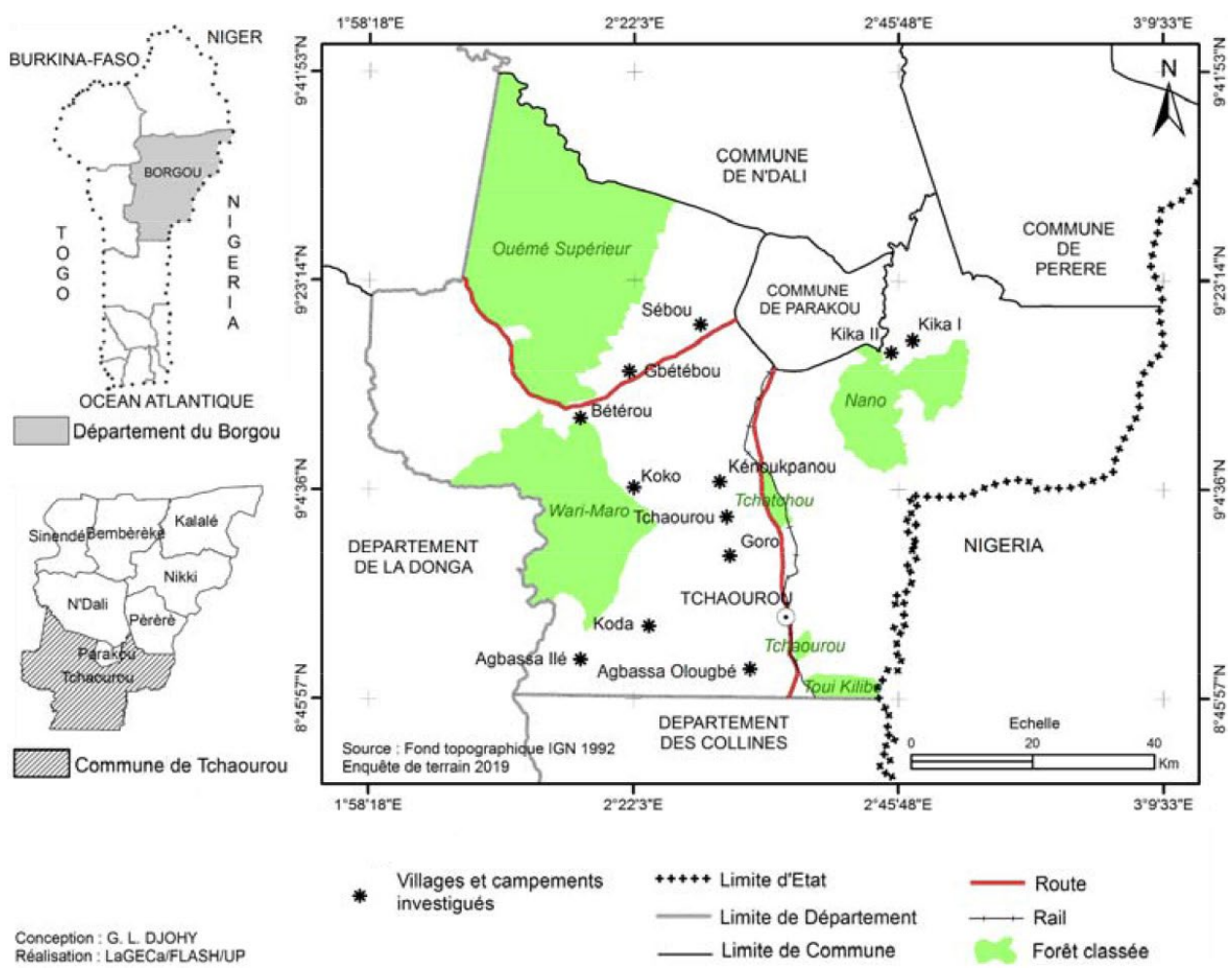

Figure 1 : Sites des enquêtes menées auprès d'agropasteurs à Tchaourou au Bénin ; Source : IGN, 1992 /// Sites of the surveys conducted with agropastoralists in Tchaourou, Benin; Source: IGN, 1992 
Les termes agropasteur ou éleveur (ce dernier terme est utilisé seul par la suite pour une lecture plus homogène) désignent cette catégorie d'acteurs ayant l'élevage bovin comme principale activité à laquelle ils associent l'agriculture comme source alternative de vie, intégrée à leur pratique d'élevage. A Tchaourou cette population est peule. C'est la raison fondamentale du choix de ces acteurs pour conduire l'étude. Les modes d'accès à la terre de production des Peuls sont l'héritage, le don, l'achat, le prêt et la location, l'héritage étant le mode d'accès le plus courant dans le département du Borgou (De Haan et al., 1997). Les autres groupes socioculturels de la commune pratiquent la polyculture-élevage. Ils développent une diversité de cultures et au moins un élevage, celui de bovins, d'ovins, de caprins ou de volailles.

\section{Collecte des données}

L'approche méthodologique s'est reposée sur le suivi des animaux au pâturage, les entretiens individuels et collectifs, et les observations. A partir de la méthode d'échantillonnage raisonné et 26 troupeaux suivis au pâturage, 130 éleveurs ont été interviewés entre avril et juin 2019. Quatre entretiens collectifs ont été organisés avec en moyenne sept éleveurs et ont permis de compléter et de confronter certaines informations recueillies lors des entretiens individuels. La documentation et l'enquête préparatoire ont permis d'identifier les principaux foyers de développement de l'élevage à Tchaourou. Ainsi, six arrondissements, sur les sept que compte la commune, ont été retenus pour les enquêtes de terrain : Bétérou, Tchatchou, Sanson, Alafiarou, Kika et Goro. Douze villages et campements ont été inclus dans ces arrondissements en fonction de l'abondance des ressources pastorales, de la forte présence d'éleveurs et de l'importance de leur cheptel bovin. Ces villages et campements ont été choisis au cours des différentes discussions menées avec les autorités villageoises et les responsables de la population peule. L'échantillon a tenu compte de critères comme l'âge et le nombre d'années d'expérience. Ainsi, les éleveurs avaient au moins 30 ans et pratiquaient l'agropastoralisme depuis au moins 10 ans. Les données collectées sur le terrain ont été relatives à leur perception de l'évolution des paramètres climatiques, notamment la pluviométrie, la température et la vitesse du vent. Les enquêtes de terrain ont permis également de collecter des données sur la vulnérabilité des différentes catégories d'éleveurs, des ressources fourragères et hydriques aux modifications climatiques, et sur les stratégies d'alimentation, d'abreuvement et de gestion des pathologies bovines.

\section{Analyse des données de terrain}

Les entretiens individuels et collectifs ont permis de mobiliser auprès des éleveurs des données qualitatives qui ont servi à l'analyse de discours (Igalens, 2007 ; Seignour, 2011). Cette méthode d'analyse des données qualitatives des sciences sociales et humaines a permis d'appréhender les perceptions des éleveurs par rapport aux mutations climatiques, leur vulnérabilité aux risques climatiques et leurs stratégies d'adaptation. La matrice de sensibilité a été réalisée suivant la technique de réalisation des matrices de vulnérabilité (ENDA et C3D, 2005). Elle a permis, d'une part, de calculer les indices d'exposition des ressources pastorales (fourragères et hydriques) et des éleveurs aux mutations climatiques, et, d'autre part, le niveau des impacts engendrés par les différents risques climatiques identifiés dans le milieu d'étude. Au cours des entretiens, les éleveurs ont décrit et noté leur ressenti au quotidien (1 faible, 2 assez faible, 3 moyen, 4 assez fort, 5 fort) vis-à-vis des modifications climatiques. De plus, ils ont décrit et noté l'efficacité (1 forte, 2 moyenne, 3 faible) variable dans le temps et dans l'espace des différentes sources d'alimentation et d'abreuvement des animaux. Les différentes notes attribuées par les éleveurs ont permis de calculer l'efficacité des ressources et des modes de traitement. L'efficacité a été considérée comme un indicateur technique de mesure du temps de disponibilité des ressources pastorales pendant la saison sèche. Elle désignait également le degré de satisfaction des éleveurs à la fin des traitements vétérinaires ou ethnovétérinaires.

Les entretiens individuels et collectifs constituent des méthodes d'enquête par excellence pour recueillir les ressentis des éleveurs sur l'efficacité des différents modes de traitement des animaux (Barbarin, 2019) et des différentes sources d'alimentation des animaux. Ainsi, les éleveurs ont exprimé leur ressenti sur l'efficacité des différentes sources d'alimentation et d'abreuvement des animaux suivant la durée de la disponibilité des ressources au cours de la saison sèche entre novembre et avril. Les ressources à faible efficacité étaient les ressources disponibles sur une période d'un à deux mois après la saison des pluies (novembre et décembre). Les ressources à efficacité moyenne et forte étaient les ressources disponibles respectivement de novembre à février et de novembre à avril. De plus, l'expression des ressentis des éleveurs a permis de caractériser leur degré de satisfaction vis-à-vis des différents modes de traitement des animaux. Les éleveurs ont qualifié de forte efficacité le mode de traitement permettant à l'animal malade de guérir, d'efficacité moyenne le mode de traitement procurant une guérison partielle et d'efficacité faible le mode de traitement n'apportant qu'une guérison momentanée. Le diagramme de vulnérabilité a été réalisé suivant un gradient international d'évaluation de vulnérabilité de 20 à 100, exprimant la facilité d'accès des différentes catégories d'éleveurs aux ressources pastorales (Djohy et al., 2017).

\section{RESULTATS}

\section{Perception des mutations climatiques par les éleveurs}

Pour les éleveurs les différents paramètres climatiques ont connu une perturbation ces dernières années qui a fortement influencé la disponibilité des ressources pour le développement de l'activité pastorale. Les mutations climatiques étaient perçues par les vents violents (98\% des éleveurs), la hausse des températures (97\%), le début tardif de la saison des pluies $(97 \%)$, le prolongement de la saison sèche (96\%), la fin précoce de la saison des pluies (94\%) et la baisse pluviométrique (90\%).

Pour $28 \%$ des éleveurs, les effets nuisibles des mutations climatiques ont été amplifiés ces dernières années par les activités anthropiques, notamment les activités agricoles et l'exploitation forestière. Selon eux les ressources pastorales ont été fortement impactées par les mutations climatiques, notamment l'assèchement précoce des points d'eau et l'amenuisement du disponible fourrager. Ces modifications climatiques ont énormément contribué à la dégradation de l'état sanitaire des animaux par le développement de diverses pathologies, dont la fièvre aphteuse, la pasteurellose bovine, la trypanosomose et la brucellose.

\section{Traits distinctifs des groupes d'éleveurs}

La taille du troupeau bovin déterminait le statut social des éleveurs au sein de la communauté pastorale et était source de prestige. Elle comptait davantage que celle du cheptel de petits ruminants et, avec la santé des bovins, constituait une préoccupation majeure pour les Peuls qui considéraient les bovins plus importants que d'autres biens, notamment les moyens de transport et les maisons. La taille du cheptel bovin déterminait également la forme de mobilité pratiquée par l'éleveur dans l'espace et le temps. Trois grandes catégories d'éleveurs se sont dégagées à partir de ces indicateurs (tableau I).

La taille du cheptel bovin constituait non seulement le fondement des systèmes de valeurs dans la communauté peule mais déterminait également les différentes pratiques pastorales ainsi que les ressources exploitées. Ainsi, la dynamique saisonnière des petits éleveurs se limitait aux espaces environnant leur territoire d'attache. En revanche, les moyens et les gros éleveurs pratiquaient une dynamique 
saisonnière à l'intérieur et à l'extérieur de leur territoire d'attache pour mieux subvenir aux besoins alimentaires du cheptel. La pratique de division du cheptel en plusieurs sous-troupeaux permettait aux gros éleveurs de mieux exploiter les ressources dispersées.

\section{Sensibilité des ressources pastorales}

Pour les personnes interviewées, l'élevage était très vulnérable à la sécheresse, à la chaleur excessive, aux pluies tardives et fortes, aux vents $\sim$ violents et aux feux de végétation. Ces risques impactaient les ressources fourragères et hydriques (tableau II) ainsi que les éleveurs (tableau III). Ils rendaient plus difficile le développement de l'activité pastorale.

Le tableau II révèle que les ressources en eau superficielle étaient, selon la perception des éleveurs, plus exposées aux risques climatiques.
Les mares, les barrages et les retenues d'eau étaient les plus exposés (indicateur d'exposition $72 \%$ ), venaient ensuite les eaux de l'Ouémé et de l'Okpara (56\%). Quant aux ressources en eau souterraine, les puits étaient exposés (44\%) et les forages ( $40 \%$ ). Les ressources fourragères, notamment les fourrages herbacés et les ligneux fourragés, étaient les plus exposées (respectivement $84 \%$ et $68 \%$ ).

Le tableau III révèle que les gros éleveurs étaient les plus exposés aux menaces climatiques ( $84 \%$ ), et les éleveurs disposant de troupeaux moyens et les petits éleveurs l'étaient mais dans une moindre mesure (respectivement $68 \%$ et $60 \%$ ). La sécheresse était le premier risque climatique qui affectait les éleveurs (indicateur d'impact $87 \%$ ), suivi des feux de végétation affectant la production animale (80\%), de la chaleur excessive $(73 \%)$, des pluies tardives et fortes $(60 \%)$ et des vents violents $(53 \%)$.

Tableau I : Typologie des agropasteurs de la commune de Tchaourou au Bénin en 2019 /// Typology of agropastoralists in the commune of Tchaourou in Benin in 2019

\begin{tabular}{|c|c|c|c|}
\hline Catégorie & Taille du cheptel & Mobilité pastorale & Ressources fourragère et hydrique \\
\hline Petit éleveur & $\begin{array}{l}20-50 \text { bovins } \\
5-30 \text { ovins }\end{array}$ & $\begin{array}{l}\text { A l'intérieur du territoire } \\
\text { d'attache }\end{array}$ & $\begin{array}{l}\text { Fourrage herbacé, ligneux fourrager et résidu de récolte } \\
\text { Eaux superficielle et souterraine }\end{array}$ \\
\hline $\begin{array}{l}\text { Moyen } \\
\text { éleveur }\end{array}$ & $\begin{array}{l}50-100 \text { bovins } \\
30-80 \text { ovins }\end{array}$ & $\begin{array}{l}\text { A l'intérieur et à l'extérieur } \\
\text { du territoire d'attache }\end{array}$ & $\begin{array}{l}\text { Fourrage herbacé, ligneux fourrager et résidu de récolte } \\
\text { Eaux superficielle et souterraine }\end{array}$ \\
\hline Gros éleveur & $\begin{array}{l}>100 \text { bovins } \\
>80 \text { ovins }\end{array}$ & $\begin{array}{l}\text { A l'intérieur et à l'extérieur } \\
\text { du territoire d'attache }\end{array}$ & $\begin{array}{l}\text { Fourrage herbacé, résidu de récolte, ligneux fourrager, } \\
\text { végétation des zones de décrues } \\
\text { Eau superficielle }\end{array}$ \\
\hline
\end{tabular}

Tableau II : Matrice de sensibilité des ressources aux risques climatiques, commune de Tchaourou au Bénin en 2019 /// Matrix of resource sensitivity to climate risks, Tchaourou Commune in Benin in 2019

\begin{tabular}{|c|c|c|c|c|c|c|c|}
\hline \multirow{2}{*}{\multicolumn{2}{|c|}{ Ressource pastorale }} & \multicolumn{5}{|c|}{ Risque climatique } & \multirow{2}{*}{$\begin{array}{c}\text { Indicateur } \\
\text { d'exposition } \\
(\%)\end{array}$} \\
\hline & & Chaleur excessive & Sécheresse & Vent violent & Pluie tardive et forte & Feu de végétation & \\
\hline \multirow{2}{*}{$\begin{array}{l}\text { Eau } \\
\text { superficielle }\end{array}$} & Fleuve et rivière & 4 & 4 & 1 & 4 & 1 & 56 \\
\hline & $\begin{array}{l}\text { Mare, barrage et } \\
\text { retenue d'eau }\end{array}$ & 5 & 5 & 2 & 4 & 2 & 72 \\
\hline \multirow{2}{*}{$\begin{array}{l}\text { Eau } \\
\text { souterraine }\end{array}$} & Puits & 3 & 3 & 1 & 3 & 1 & 44 \\
\hline & Forage & 3 & 3 & 1 & 2 & 1 & 40 \\
\hline \multirow{3}{*}{$\begin{array}{l}\text { Ressource } \\
\text { fourragère }\end{array}$} & Fourrage herbacé & 4 & 5 & 3 & 4 & 5 & 84 \\
\hline & Ligneux fourrage & 3 & 3 & 4 & 3 & 4 & 68 \\
\hline & Résidu agricole & 2 & 3 & 2 & 2 & 5 & 56 \\
\hline
\end{tabular}

1 faible, 2 assez faible, 3 moyenne, 4 assez forte, 5 forte /// 1 low, 2 medium low, 3 intermediate, 4 medium high, 5 high

Tableau III : Matrice de sensibilité des agropasteurs aux risques climatiques, commune de Tchaourou au Bénin en 2019 /// Sensitivity matrix of agropastoralists to climatic risks, Tchaourou Commune in Benin in 2019

\begin{tabular}{|c|c|c|c|c|c|c|}
\hline \multirow[t]{2}{*}{ Eleveur } & \multicolumn{5}{|c|}{ Risque climatique } & \multirow{2}{*}{$\begin{array}{l}\text { Indicateur } \\
\text { d'impact } \\
(\%)\end{array}$} \\
\hline & Chaleur excessive & Sécheresse & Pluie tardive et forte & Vent violent & Feu de végétation & \\
\hline Petit éleveur & 3 & 4 & 2 & 3 & 3 & 60 \\
\hline Moyen éleveur & 3 & 4 & 3 & 3 & 4 & 68 \\
\hline Gros éleveur & 5 & 5 & 4 & 2 & 5 & 84 \\
\hline Indicateur d'impact (\%) & 73 & 87 & 60 & 53 & 80 & - \\
\hline
\end{tabular}

1 faible, 2 assez faible, 3 moyenne, 4 assez forte, 5 forte /// 1 low, 2 medium low, 3 intermediate, 4 medium high, 5 high 


\section{Vulnérabilité des groupes d'éleveurs}

Le niveau d'accès des différentes catégories d'éleveurs aux ressources pastorales disponibles a permis de réaliser leur profil de vulnérabilité (figure 2). Il révèle que les gros éleveurs étaient plus vulnérables aux effets nuisibles des changements et variabilités climatiques que les moyens et les petits éleveurs car ils avaient un accès relativement restreint aux différentes ressources pastorales. La répartition du cheptel en sous-troupeaux devenait une nécessité non seulement pour faciliter l'entretien du troupeau mais aussi pour assurer l'alimentation et l'abreuvement des animaux en saison sèche. Pour faire face à cette vulnérabilité, les éleveurs développaient diverses stratégies qui leur permettaient d'atténuer les effets des changements climatiques sur leur activité.
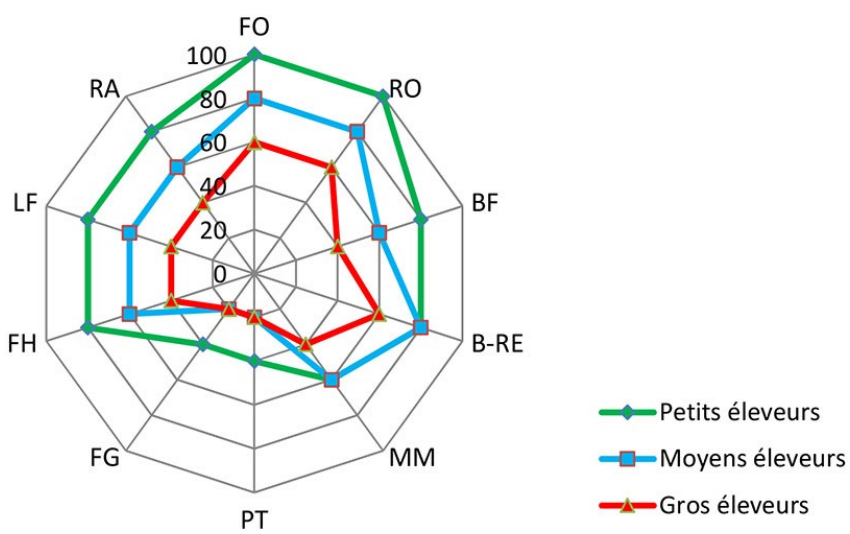

Figure 2 : Profil de vulnérabilité des agropasteurs à Tchaourou au Bénin en 2019 ; FO : fleuve Ouémé ; RO : rivière Okpara ; $\mathrm{BF}$ : bas-fonds ; B-RE : barrages et retenues d'eau ; MM : mares et marigots ; PT : puits ; FG : forages ; FH : fourrage herbacé ; LF : ligneux fourragers; RA : résidus agricoles /// Vulnerability profile of agropastoralists in Tchaourou, Benin in 2019; FO: Ouémé River; RO: Okpara River; BF: lowlands; B-RE: dams and water reservoirs; MM: ponds; PT: wells; FG: boreholes; FH: herbaceous fodder; LF: woody fodder; RA: agricultural residues

\section{Stratégies d'adaptation}

Les stratégies d'adaptation développées par les éleveurs face aux nouvelles conditions climatiques étaient de plusieurs ordres. Elles englobaient les différentes pratiques de gestion de l'eau, des ressources fourragères et des différentes pathologies.

\section{Sources d'abreuvement des animaux}

Pour répondre aux besoins en eau du bétail, les éleveurs ont diversifié les sources d'abreuvement (figure 3 à gauche). Pendant la saison sèche, les sources d'eau permanentes (fleuve, rivière, barrages, retenues d'eau) étaient très fréquentées tandis que les sources d'eau temporaires l'étaient moins à cause de leur assèchement rapide. Les éleveurs avaient ainsi recours aux retenues d'eau (33\%), aux surcreusements des bas-fonds (24,8\%), aux barrages (14,3\%), au fleuve Ouémé (12\%) et aux forages (12\%). Les sources d'abreuvement des animaux ont présenté une efficacité variable dans le temps (figure 3 à droite), l'efficacité désignant la durée de la disponibilité des ressources en eau pendant la saison sèche. Les points de surcreusement des basfonds tarissaient entre novembre et décembre et ne permettaient pas aux éleveurs d'abreuver les animaux. Ces sources présentaient une faible efficacité selon les enquêtes. Seuls le fleuve, les retenues d'eau et les barrages ont présenté une forte ou une moyenne efficacité et ont permis aux éleveurs d'abreuver les animaux en saison sèche.

\section{Sources d'alimentation des animaux}

Le manque de fourrage herbacé en saison sèche rendant l'alimentation des animaux difficile, les éleveurs ont développé différentes techniques pour les nourrir (figure 4 à gauche). L'exploitation des résidus de récolte (42\%), des ligneux fourragers (42\%) et des végétations des zones humides et forêts $(10 \%)$ ont constitué les principales stratégies développées par les éleveurs pour mieux nourrir le bétail en saison sèche. L'efficacité de ces stratégies a varié dans le temps (figure 4 à droite). Les ligneux fourragers, les végétations des zones humides et des forêts ont eu une grande efficacité en saison sèche. La culture fourragère fraîche de Panicum maximum $\mathrm{C} 1$ convenant au bétail, les petits et moyens éleveurs de Tchaourou avaient commencé
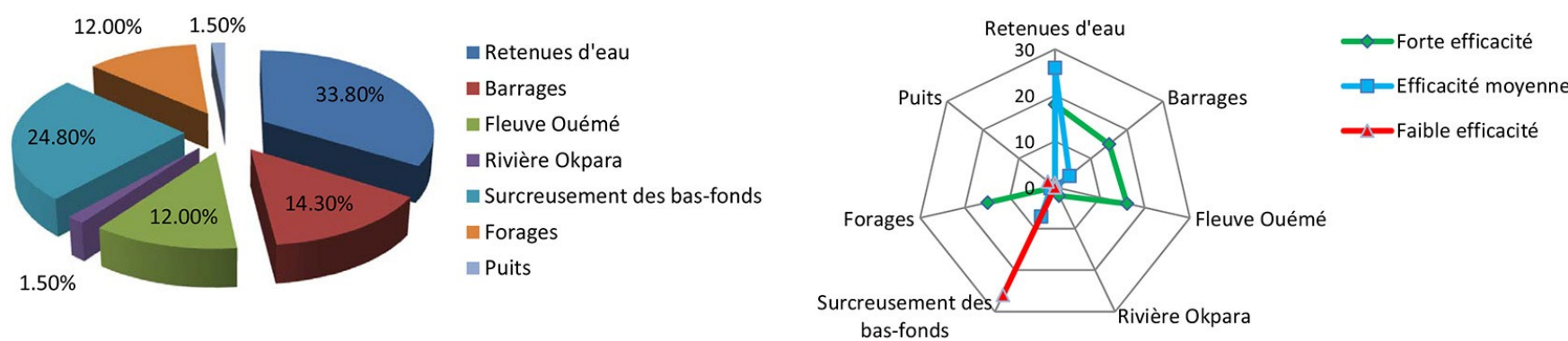

Figure 3 : Sources d'abreuvement du bétail (gauche) et leur efficacité (droite) à Tchaourou au Bénin en 2019 /// Livestock drinking sources (left) and their efficiency (right) in Tchaourou, Benin in 2019

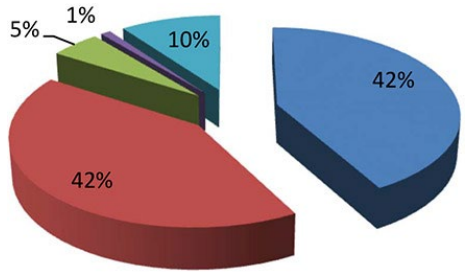

- Ligneux fourragers

nésidus agricoles

nelures de manioc et d'igname

n Culture fourragère

- Végétation zones humides et forêt
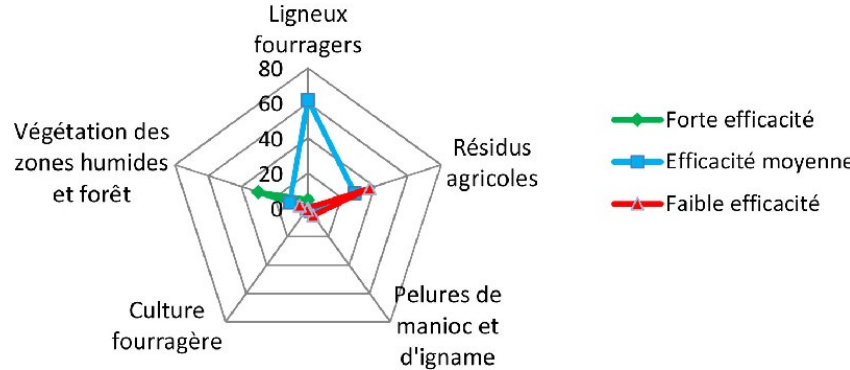

- -Faible efficacité

Figure 4 : Sources d'alimentation du bétail (gauche) et leur efficacité (droite) à Tchaourou au Bénin en 2019 /// Livestock feed sources (left) and their efficiency (right) in Tchaourou, Benin in 2019 
à la développer (figure 5). Ainsi depuis 2017, dans le cadre du projet de «Contrôle de l'invasion de Hyptis suaveolens Poit. dans les pâturages naturels par la culture de $P$. maximum $\mathrm{Cl}$ dans les exploitations peules au Bénin », les éleveurs de la commune ont pu installer des parcelles d'essai de plus de 500 kilomètres carrés. Par ailleurs, tous les éleveurs utilisaient les résidus de récolte notamment de maïs, de riz, de mil/sorgho, et les pelures de manioc et d'igname en complément pour nourrir le bétail.

\section{Gestion des pathologies bovines}

La santé des animaux est menacée par les nouvelles conditions environnementales et climatiques. Les éleveurs ont développé diverses stratégies pour lutter contre les pathologies des animaux notamment la fièvre aphteuse, la péripneumonie contagieuse bovine, la pasteurellose bovine, la trypanosomose, la tuberculose et la brucellose. Le traitement vétérinaire $(55 \%)$ et le traitement ethnovétérinaire (43\%) ont constitué leurs principales stratégies. Cependant, l'efficacité des différents traitements a varié en fonction de la pathologie. Le traitement mixte consistait à consulter un vétérinaire, puis à administrer à l'animal une combinaison de produits pharmaceutiques et traditionnels. Le traitement vétérinaire a présenté une forte efficacité selon les éleveurs et le traitement ethnovétérinaire a eu une efficacité moyenne. Il a cependant été très efficace dans le traitement des infections nasales et des morsures de serpent.

\section{DISCUSSION}

\section{Perception du changement climatique}

Les éleveurs percevaient les mutations climatiques à travers plusieurs indicateurs, dont la hausse des températures, la mauvaise répartition spatio-temporelle des pluies et les vents violents. Ces résultats étaient similaires à ceux obtenus par Ouedraogo (2010), Kate et al. (2015), Djohy et al. (2017), Dayamba et al. (2019), Abdou et al. (2020) et Idrissou et al. (2020). Pour Boko et al. (2012) et Djohy (2015), la région septentrionale du Bénin enregistre des périodes excédentaires et déficitaires qui sont dues à des phénomènes exceptionnels, comme les sécheresses et les excédents pluviométriques. Ainsi, le Nord Bénin connaît une hausse des températures de $+0,5$ à $1^{\circ} \mathrm{C}$, des cas d'extrêmes pluviométriques et une sécheresse généralisée (Boko et al., 2012). Les changements et variabilités thermométriques de surface indiquent un réchauffement climatique de $0,85[0,65-1,06]^{\circ} \mathrm{C}$ (GIEC, 2013). Cette hausse s'accompagne d'une diminution significative de la fréquence des jours très froids et d'une augmentation de la fréquence des jours très chauds (Ly et al., 2013). Les incidences d'événements climatiques extrêmes (canicule, sécheresse, inondation) mettent en évidence la vulnérabilité et le niveau élevé d'exposition des écosystèmes et des systèmes humains aux changements climatiques (GIEC, 2014). Ces évolutions du climat influencent la disponibilité des ressources pastorales et rendent vulnérables les différents systèmes pastoraux.

\section{Vulnérabilité des ressources et des éleveurs aux mutations climatiques}

Les ressources fourragères et hydriques et les différentes catégories d'éleveurs de la commune sont menacées par les risques climatiques. Ces résultats corroborent ceux de Kate et al. (2015), Djohy et al. (2017) et Abdou et al. (2020) qui montrent que l'incertitude climatique constitue un facteur important de vulnérabilité pour les ressources pastorales et les éleveurs. Pour Tente et al. (2013) et Alwaly (2017), la vulnérabilité pastorale s'exprime dans un premier temps par la dégradation des conditions de survivance du bétail, notamment le manque de pâturage, d'eau pour l'abreuvement et les risques épidémiologiques. Elle s'exprime dans un second temps par la confrontation des éleveurs à une multitude de chocs notamment les sécheresses, les phénomènes d'inondations, l'appropriation des terres, les actes de banditisme, le terrorisme et les pathologies animales (Alwaly, 2017). La baisse du cumul pluviométrique et la hausse des températures impactent la disponibilité des ressources (Sanogo et al., 2016). Pour Manoli (2012), Kate et al. (2015), la vulnérabilité des éleveurs est préoccupante face aux changements globaux notamment climatiques. Ainsi, la dégradation des aires de pâturage, la raréfaction des points d'abreuvement des animaux, la détérioration de leur état sanitaire et la fréquence des sécheresses constituent des indicateurs qui attestent la vulnérabilité des ressources aux changements climatiques et environnementaux et interpellent les éleveurs sur l'urgence de développer des stratégies appropriées (Dayamba et al., 2019 ; Abdou et al., 2020).

\section{Stratégies d'adaptation}

Face à la réduction des ressources fourragères et hydriques en saison sèche, les éleveurs de Tchaourou ont développé diverses stratégies pour nourrir et abreuver les animaux. Ces stratégies consistaient à utiliser les résidus de récolte, les ligneux fourragers et la végétation des zones humides comme rapporté par Djènontin (2010), Djohy (2015), Dayamba et al. (2019) et Abdou et al. (2020). Si en saison pluvieuse le fourrage herbacé est abondant et peut couvrir les besoins alimentaires des animaux,
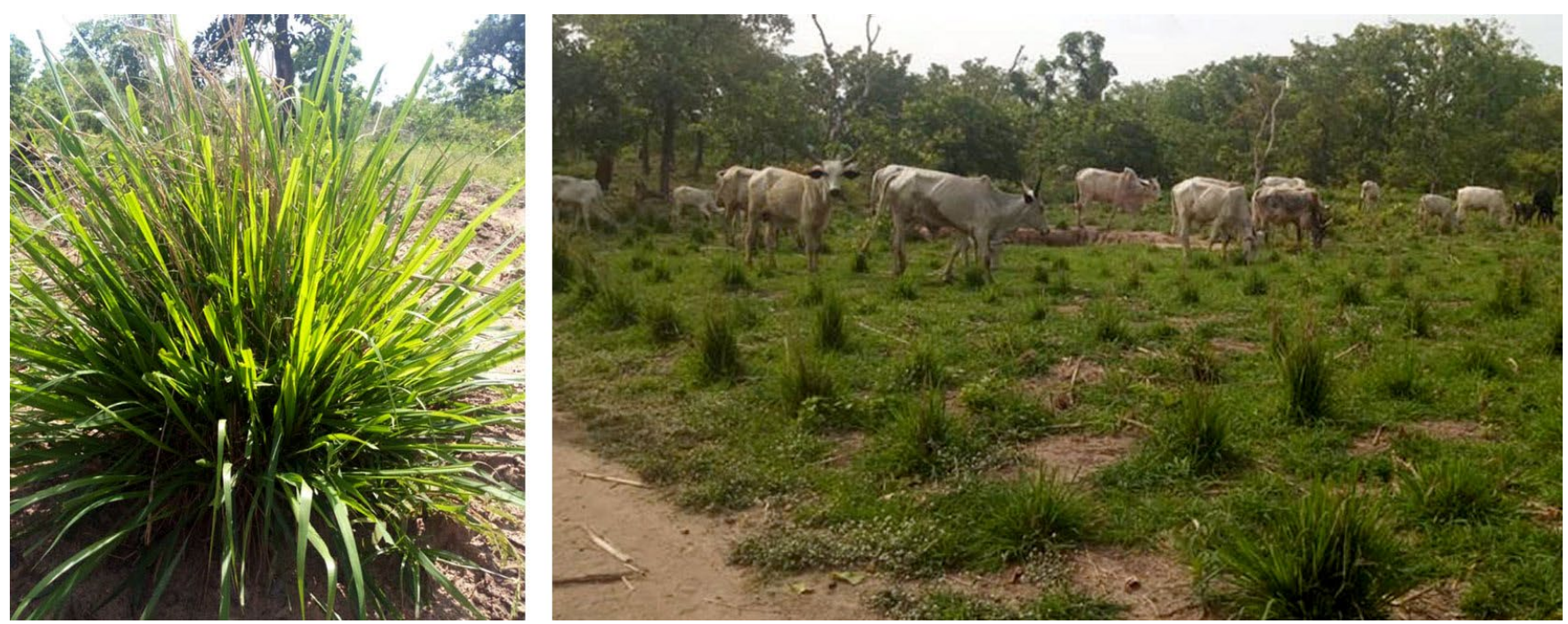

Figure 5 : Culture fourragère de Panicum maximum C1 à Tchaourou au Bénin en 2019 ; (C) Djohy G.L. /// Fodder crop of Panicum maximum C1 at Tchaourou in Benin in 2019; ( D Djohy G.L. 
en saison sèche il devient rare (Djènontin et al., 2009). Pour Salifou (2010) la production fourragère sans les résidus culturaux ne couvre les besoins alimentaires des animaux qu'une année sur trois voire quatre. L'exploitation des résidus culturaux par les animaux constitue un enjeu stratégique pour les éleveurs et les agropasteurs (Touré, 2015). La pérennité du système pastoral repose aussi sur l'accès à l'eau du troupeau. Ainsi, en saison sèche les éleveurs avaient recours à diverses sources d'eau souterraines ou de surface, à l'instar des résultats obtenus par Cornu et al. (2008) qui rapportent par ailleurs que le fonçage ou la réhabilitation de puits pastoraux et le surcreusement des mares constituent des stratégies des éleveurs pour faire face à la baisse des ressources en eau en saison sèche. Le faible accès aux ressources pastorales expose les animaux à diverses pathologies (Simel, 2010). Dans ces conditions, les éleveurs ont combiné divers produits dans le traitement des animaux, notamment vétérinaires et ethnovétérinaires. Les Peuls ont souvent recours aux plantes médicinales (Byavu et al., 2000) et aux méthodes modernes (Abdou et al., 2020) comme les antibiotiques (Mensah et al., 2014 ; Vieira, 2019) et les trypanocides (Dia et Desquesnes, 2003) dans le traitement des différentes pathologies bovines.

Les variations climatiques et environnementales ont contraint les éleveurs à pratiquer une diversité de mobilités pastorales à la recherche des ressources fourragères et hydriques. Ces mouvements ont constitué des stratégies d'adaptation en réponse à la sévérité des dérèglements climatiques et à la variabilité spatio-temporelle des écosystèmes pâturés (Djènontin, 2010 ; Djohy, 2015). La mobilité pastorale suit un calendrier bien établi en fonction de la variation écologique pour permettre aux pasteurs d'exploiter les ressources dispersées. Elle est régie par des textes régionaux et nationaux. Ainsi, la Communauté économique des Etats de l'Afrique de l'Ouest (Cedeao) a adopté à Abuja le 31 octobre 1998 la décision A/DEC.5/10/98 relative à la règlementation de la mobilité pastorale entre ses Etats membres, instaurant un premier instrument juridique destiné à sécuriser les conditions de déplacement et d'accueil du bétail transhumant. Ces Etats ont adopté également des textes pour réglementer la mobilité au plan national. C'est le cas du Bénin qui a adopté plusieurs textes législatifs et réglementaires pour une gestion apaisée de la mobilité (Yahaya Namassa, 2014). Le 21 septembre 1987 il a adopté la « loi n 87-013 portant réglementation de la vaine pâture, de la garde des animaux domestiques et de la transhumance ». Des arrêtés interministériels ont également été pris dans le but de sécuriser la mobilité des pasteurs. Toutefois, le non-respect de ces lois a conduit le gouvernement béninois à prendre le 13 décembre 2017 des mesures restrictives par rapport à la mobilité nationale et transfrontalière. Il a ensuite adopté la « loi n 2018-20 du 23 avril 2019 portant code pastoral en République du Bénin ». Malgré les différents textes régionaux et nationaux, la mobilité pastorale demeure une source de conflits et de tensions entre usagers des ressources naturelles (FAO, 2012 ; Yahaya Namassa, 2014). Déplorant de nombreuses pertes humaines et des dégâts matériels, l'arrêté interministériel béninois du 26 décembre 2019 restreint la mobilité nationale et interdit la mobilité transfrontalière. Dès lors, le gouvernement s'est lancé dans la

\section{REFERENCES}

Abdou H., Karimou I.A., Harouna B.K., Zataou M.T., 2020. Perception of climate change among Sahelian pastoralists and strategies for adapting to environmental constraints: the case of the commune of Filingué in Niger. Rev. Elev. Med. Vet. Pays Trop., 73 (2): 81-90, doi: 10.19182/ remvt. 31873

Alwaly A.A.A., 2017. Entretien : que faire face à la vulnérabilité pastorale ? Grain sel, 73-74: 2 p.

Barbarin S.M.P., 2019. Etude épidémiologique de la dermatophilose et de la démodécie bovine amayotte et propositions de protocoles de traitement. Thèse doct., Ecole nationale vétérinaire d'Alfort, France, 145 p. promotion des cultures fourragères notamment du Panicum maximum $\mathrm{C} 1$ afin de sédentariser les éleveurs. Cet arrêté a contribué sans doute à accroître les difficultés d'accès des pasteurs aux ressources pastorales. La situation est si critique au niveau régional que le gouvernement a accordé, lors des négociations avec le Niger le 18 février 2020, un moratoire exceptionnel de deux mois (mars et avril) aux pasteurs nigériens pour entrer au Bénin avec leurs troupeaux. Le Bénin et le Burkina Faso ont aussi récemment envisagé une coopération transfrontalière à travers un accord-cadre validé lors d'un atelier bilatéral à Cotonou (Mehouenou, 2020), dont la mise en œuvre pourrait aussi profiter au secteur de la mobilité transfrontalière de bétail.

\section{CONCLUSION}

L'approche utilisée dans cette étude a mis en évidence la perception des mutations climatiques par les éleveurs, leur vulnérabilité aux risques climatiques et leurs stratégies d'adaptation aux nouvelles conditions climatiques et environnementales. Les résultats montrent qu'ils sont davantage menacés par le dérèglement climatique, en l'occurrence le déficit pluviométrique, la fin précoce de la saison des pluies, la hausse des températures et les vents violents. Pour eux, les phénomènes de hausse des températures s'observent pendant les périodes sèches. Quant aux vents violents, ils estiment qu'ils s'observent toute l'année mais sont plus fréquents pendant la saison des pluies. Ces modifications climatiques rendent l'activité agropastorale très vulnérable. Elles entraînent la raréfaction des ressources pastorales et la dégradation de l'état sanitaire des animaux. Face à la dynamique environnementale et climatique, les éleveurs ont recours à diverses sources d'eau souterraines ou de surface pour abreuver des animaux. L'alimentation des animaux est rendue possible grâce à la combinaison des résidus de récolte, des ligneux fourragers et de la végétation des bas-fonds. L'association des traitements vétérinaires et ethnovétérinaires permet aux éleveurs de mieux lutter contre les pathologies et les épidémies animales. L'analyse des stratégies adoptées montre qu'elles sont pertinentes au regard de leur perception. Ces mesures d'adaptation leur permettent de continuer à développer leur activité agropastorale et à sécuriser leurs moyens d'existence dans un environnement de plus en plus incertain. Par ailleurs, il est urgent de développer des politiques publiques plus sensibles aux mutations climatiques et à la gestion des ressources pastorales afin de renforcer les capacités adaptatives des éleveurs.

\section{Déclaration des contributions des auteurs}

GLD et BSB ont conçu et planifié l'étude ; GLD a collecté les données, effectué les analyses statistiques et rédigé le manuscrit ; GLD et BSB ont révisé toutes les versions du manuscrit.

\section{Conflits d'intérêts}

L'étude a été réalisée sans conflit d'intérêts.

Boko M., Kosmowski F., Vissin W.E., 2012. Les enjeux du changement climatique au Bénin : Programme pour le dialogue politique en Afrique de I'Ouest. Konrad-Adenauer-Stiftung, Cotonou, Bénin, 65 p.

Byavu N., Henrard C., Dubois M., François M., 2000. Phytothérapie traditionnelle des bovins dans les élevages de la plaine de la Ruzizi. Biotechnol. Agron. Soc. Environ., 4 (3): 135-156

Chabi Boum O.B.M., 2011. Pastoralisme dans la Commune de Tchaourou Organisations, Contraintes et Incidences Environnementales. Mém. maîtrise Géographie, Université d'Abomey-Calavi, Cotonou, Bénin, 87 p.

Cornu F., Chapon M., Larbodière L., 2008. La sécurisation des systèmes fonciers au Mali. Gestion concertée des ressources pastorales et sécurisation du petit élevage, Projet de sécurisation de l'élevage (PROSEL). Agronomes et vétérinaires sans frontières, $14 \mathrm{p}$. 
CSAO/OCDE, 2008. Notes aux décideurs n 3, Promouvoir et accompagner la transformation du pastoralisme transhumant dans les pays du Sahel et de I'Afrique de l'Ouest, $4 \mathrm{p}$.

Dayamba S.D., D’haen S., Coulibaly O.J.D., Korahiré J.A., 2019. Etude de la vulnérabilité des systèmes de production agro-sylvo-pastoraux face aux changements climatiques dans les provinces du Houet et du Tuy au Burkina Faso. Climate Analytics, gGmbH, Berlin, 46 p.

De Haan L.J., Biaou G., Fanou J., 1997. Le contexte national et régional 19-34. In : De Haan L.J., Agriculteurs et éleveurs au Nord Bénin. Ecologie et genre de vie, Karthala, Paris, France, 217 p.

Dia M.L., Desquesnes M., 2003. Utilisation rationnelle des trypanocides. Fiche technique ${ }^{\circ} 3$, CIRDES, Burkina Faso, CIRAD, France, 7 p.

Djènontin A.J., 2010. Dynamique des stratégies et des pratiques d'utilisation des parcours naturels pour l'alimentation des troupeaux bovins au Nord -est du Bénin. Thèse Doct., Université d'Abomey-Calavi, Cotonou, Bénin, 274 p.

Djènontin P.J.A., Houinato M., Toutain B., Sinsin B., 2009. Pratiques et stratégies des éleveurs face à la réduction de l'offre fourragère au Nord-est du Bénin. Sécheresse, 20 (4): 346-353, doi: 10.1684/sec.2009.0204

Djohy G., Edja A.H., Djenontin A.J., Houinato M., 2017. Vulnérabilité et dynamiques sociopolitiques d'adaptation des éleveurs transhumants aux perturbations climatiques au Nord du Bénin. In: Les systèmes socio-écologiques en Afrique du nord et de l'ouest face au changement global. Gouvernance, Adaptation, Viabilité et Résilience, CERES, Agadir, Maroc, 260-276

Djohy G.L., 2015. Rythme climatique et activités pastorales dans la commune de Sinendé. Mém. géographie, Université de Parakou, Bénin, 57 p.

ENDA / C3D, 2005. Compte rendu de l'atelier de formation de formateurs sur vulnérabilité et adaptation aux changements climatiques, ENDA/C3D, Dakar, Sénégal, 26 p.

FAO, 2012. La transhumance transfrontalière en Afrique de l'Ouest, Proposition de plan d'action. FAO, Rome, Italie, $146 \mathrm{p}$.

GIEC, 2001. Bilan 2001 des changements climatiques, Conséquences, adaptation et vulnérabilité. GIEC, Genève, Suisse, 97 p.

GIEC, 2013. Changements climatiques 2013 : Les éléments scientifiques. Résumé à l'intention des décideurs. OMM / PNUE, Genève, Suisse, 204 p.

GIEC, 2014. Changements climatiques 2014 : Incidences, adaptation et vulnérabilité. Résumé à l'intention des décideurs. OMM, Genève, Suisse, $34 \mathrm{p}$.

Hounkponou K.S., Bokonon-Ganta E., Nouatin G., Gnangassi C., Ahounou M., 2009. Changements climatiques au Bénin : Vulnérabilité et stratégies d'adaptation, Agridape, 24 (4) : 8-10

Idrissou Y., Seidou A.A., Tossou F. M., Worogo H.S.S., Baco M.N., Adjassin J.S., Assogba B.G.C., et al., 2020. Perception du changement climatique par les éleveurs de bovins des zones tropicales sèche et subhumide du Bénin : comparaison avec les données météorologiques. Cah. Agric., 29 (1): 1-9, doi : 10.1051/cagri/2019032

Igalens J., 2007. L'analyse du discours de la RSE à travers les rapports de développement durable. Rev. Finance-Contrôle-Stratégie, 129-155

Kate S., Amagnide A., Hounmenou C.G., Hounkpatin E.L.B., Sinsin B., 2015. Changements climatiques et gestion des ressources pastorales en zone agropastorale au Nord Bénin : cas de la commune de Banikoara. Afr. Sci., 11 (4): 201-215
Kora O., 2006. Monographie de la Commune de Tchaourou. Mission de décentralisation, Bénin, Afrique Conseil, 45 p.

Ly M., Traore S.B., Alhassane A., Sarr B., 2013. Evolution of some observed climate extremes in the West African Sahel. Weather Clim. Extrem., 1: 19-25, 10.1016/j.wace.2013.07.005

Manoli C., 2012. Le troupeau et les moyens de sécurisation des campements pastoraux. Une étude de la gestion des troupeaux de la communauté rurale de Tessekre, dans le Ferlo sénégalais. Thèse Doct., SIBAGHE, Montpellier, France, $189 \mathrm{p}$.

Mehouenou J.F., 2020. Coopération transfrontalière : le Bénin et le Burkina Faso en quête d'une approche bénéfique. La Nation Bénin https://lanationbenin.info/cooperation-transfrontaliere-le-benin-et-le-burkina-faso-en-quete-dune-approche-benefique/ (consulté 25/09/2020)

Mensah S.E.P., Laurentie M., Salifou S., Sanders P., Mensah G.A., Abiola F.A., Koudandé O.D., 2014. Usage des antibiotiques par les éleveurs bovins au centre du Bénin, quels risques pour la santé publique? Bull. Rech. Agron. Bénin, 75 (1): 1-16

Ouedraogo D., 2010. Perception et adaptation des éleveurs pasteurs au changement climatique en zones sahélienne, nord et sud soudaniennes du Burkina Faso. Mém. DEA, Université Polytechnique de Bobo-Dioulasso, Burkina Faso, 55 p.

PDC, 2017. Plan de développement communal de Tchaourou. Troisième génération 2017-2021. Ministère de la Décentralisation et de la Gouvernance locale, Bénin, 168 p.

Salifou B., 2010. Contribution au forum sur la transhumance transfrontalière. Rapport général du forum sous régional sur la transhumance transfrontalière, Gogounou, Bénin, 29-37

Sanogo T., Ballo A., Garba I., 2016. Vulnérabilité des ressources pastorales face à la variabilité et au changement climatique dans la commune rurale de Tioribougou, Mali. Cah Centre Béninois Rech Sci Tec, 10: 34-59

Seignour A., 2011. Méthode d'analyse des discours : l'exemple de l'allocution d'un dirigeant d'entreprise publique. Rev. Fr. Gest., 37 : 29-45, doi: 10.3166/RFG.211.29-45

Simel O.J., 2010. Le pastoralisme et les défis du changement climatique. In : Pasteurs nomades et transhumants autochtones. L'Harmattan, Paris, France, $61-75$

Tente B.H.A., Toko Imorou I., Lougbegnon T., Boni Y., 2013. Elaboration des cartes nationales et départementales de transhumance au Bénin. Rapport final de consultation, CREDD, Bénin, $24 \mathrm{p}$.

Totin Vodounon S.H., Djohy G.L., Amoussou E., Boko M., 2016. Instabilité du régime climatique et dynamique des systèmes pastoraux dans la commune de Sinendé au Nord-Benin. Rev. Sci. Environ. Univ. Lomé, Togo, 13: 158178

Touré O., 2015. Rapport de capitalisation des modèles de sécurisation du foncier pastoral en Afrique de l'Ouest. Réseau des organisations d'éleveurs et pasteurs de l'Afrique, $34 \mathrm{p}$.

Vieira K., 2019. Utilisation des antibiotiques en élevage bovin laitier : enquête auprès des éleveurs sur les pratiques à risque. Thèse Doct., Université Claude-Bernard, Lyon I, France, 99 p.

Yahaya Namassa Z., 2014. La transhumance transfrontalière en Afrique de I'Ouest. Paix Sécur. Int., 2: 31-46, doi: 10.25267/Paix_secur_int.2014.i2.02 


\section{Summary}

Djohy G.L., Sounon Bouko B. Vulnerability and adaptive dynamics of agropastoralists to climate change in the commune of Tchaourou in Benin

Cattle breeding is the main economic activity of the Fulani communities of North-Central Benin. This activity in Tchaourou Commune, with its Southern Sudanese climate with one rainy and one dry season, is very vulnerable to the disastrous effects of climate change. The objective of this study was to assess the perceptions of agropastoralists with regard to climate change, and to analyze their vulnerability and adaptation strategies to face it. The methodological approach was based on the collection of qualitative socio-anthropological data from field surveys. Data from individual interviews, focus groups and direct observations generated sensitivity and vulnerability matrices on the resources and agropastoralists, and allowed to analyze breeders' adaptive practices. The results showed a great similarity of the forms of climate change as perceived by the agropastoralists. These forms included rising temperatures, longer dry season, early ending of the rainy season, and reduced rainfall. These climatic risks led to the early drying up of water points, a reduction in the availability of fodder and the deterioration of animal health through the development of diseases. Faced with this climate instability and its effects, the agropastoralists have developed various strategies to mitigate its impacts. These strategies have shown varied performance depending on their efficacy and should be reinforced by a sectoral policy sensitive to climate risks.

Keywords: cattle, vulnerability, climate change adaptation, Benin

\section{Resumen}

Djohy G.L., Sounon Bouko B. Vulnerabilidad y dinámicas adaptativas de los agro pastores a las mutaciones climáticas en la comunidad de Tchaourou en Benín

La cría bovina es la principal actividad económica de las comunidades peules en el centro norte de Benín. Esta actividad en la comunidad de Tchaourou con clima sud sudanés presenta una estación lluviosa y una estación seca, y es muy vulnerable a los efectos desastrosos de las mutaciones climáticas. El presente estudio tuvo por objetivo el de comprender las percepciones de los agro pastores con respecto a las mutaciones climáticas y el de analizar su vulnerabilidad y sus estrategias de adaptación para enfrentarlas. El enfoque metodológico reposó sobre la colecta de datos cualitativos socio-antropológicos a partir de encuestas de campo. Los datos de las entrevistas individuales, de grupos de discusión y de observaciones directas permitieron generar matrices de sensibilidad y de vulnerabilidad de los recursos y de los agro pastores, así como analizar las prácticas adaptativas de estos últimos. Los resultados mostraron una gran similitud de las manifestaciones de las mutaciones climáticas percibidas por los agrocriadores. Estas manifestaciones fueron, entre otras, el aumento de las temperaturas, el prolongamiento de la estación seca, el fin precoz de la estación de lluvias y la baja pluviométrica. Estos riesgos climáticos indujeron a la desecación precoz de los puntos de agua, al agotamiento del forraje disponible y a la degradación del estado sanitario de los animales mediante el desarrollo de patologías. Frente a esta inestabilidad del clima y a sus efectos, los agro pastores desarrollaron diversas estrategias para atenuar los impactos. Estas estrategias demostraron un rendimiento variado según la eficacidad y merecen ser reforzadas mediante una política sectorial sensible a los riesgos climáticos.

Palabras clave: ganado bovino, vulnerabilidad, adaptación al cambio climático, Benín 
